\title{
ALOHA: an innovative system for surveillance of hydraulic structures
}

\author{
Paul-Henri FAURE ${ }^{1, a}$, Vanina GBIORCZYK ${ }^{2}$, Violaine DEGEZELLE ${ }^{2}$, Frédéric ZENSS ${ }^{3}$ and Vincent MORISSEAU ${ }^{4}$ \\ ${ }^{1} \mathrm{CNR} \mathrm{CACOH} 4$ rue de Chalon sur Saone, Lyon, France \\ ${ }^{2}$ SHEM 1 Rue Louis Renault - BP 13383, 31133 BALMA Cedex France \\ ${ }^{3}$ CNR DSI, 2 rue André Bonin, France \\ ${ }^{4}$ Advitam Group Rueil Malmaison, France
}

\begin{abstract}
Developers and operators of a full range of hydroelectricity structures (500 kms of dykes, 19 in-stream structures and 12 high-head installations) for over 100 years, CNR and SHEM are required to guarantee a constant monitoring of their infrastructure to ensure hydraulic safety, meet their regulatory requirements and to be prepared to new challenges: improved efficiencies, optimised monitoring of high risks areas and information sharing between the different operators.

To reach a better surveillance and improve risk management, CNR and SHEM called upon the Advitam Group to set up, in less than 12 months, the innovative software solution 'ALOHA' for the inspections and monitoring of their structures to a better knowledge of risk.
\end{abstract}

This monitoring is ensured by over 15,000 sensors, allowing an analysis of historic data spread over 30 years.

ALOHA, a development based on the ScanPrint ${ }^{\circledR}$ web based software, allows the full range of operators to collect, visualise, analyse and edit all of the auscultation and monitoring data in an integrated platform. To respond to the specific needs of CNR and SHEM, innovative modules were developed for both in-office work and mobile tools.

This article will detail the functional requirements of the CNR and SHEM, the solutions proposed by Advitam with the ALOHA system and the integration of the solution in the risk management.

\section{PRESENTATION OF THE CONTEXT}

In the context of the advent of new technologies, in order to ensure the longevity of structures, and driven by exacting "safety" regulations, the surveillance tools for hydraulic structures need to undergo a transformation. The ALOHA project fits neatly in this scope, and within the ambition of the Engie Group.

As concession-holder and developer of the Rhône River since 1933, CNR operates, monitors and maintains 19 run-of-river hydroelectricity developments over an approx. $600 \mathrm{~km}$ stretch. The entire estate comprises some fifty concrete structures and $400 \mathrm{~km}$ of dikes. All themeasures and $90 \%$ of the surveillance is carried out internally by CNR's own departments, both local (Measurements teams) and support (Centre d'Analyse Comportementale des Ouvrages Hydraulique et Direction de l'Ingénierie [Behavioural Analysis Centre for Hydraulic Works and Engineering Department]). All the works are monitored by the 15,000 monitoring measured at weekly to quarterly intervals.

The Société Hydroélectrique du Midi (SHEM), operates, monitors and maintains 58 hydroelectricity plants, the majority being high-head, plus 12 dams in the Pyrenees, the Massif Central and the valley of the Lot. From the $1^{\text {st }}$ of January 2016, SHEM will carry out all examination operations on these dams internally, where some of this work has previously been outsourced; to this end, the departments concerned (the Safety Pole in the head office and on-site operators) and also the Engie experts instructed by SHEM to analyse its examination data, will use the ALOHA tool.

ALOHA was initiated in November 2013 by the CNR's Centre d'Analyse Comportementale des Ouvrages Hydrauliques and relates more specifically to the redesign of the current structural examination database. SHEM joined this project in 2014 in order to incorporate its specific needs relating to high-head and medium-head works in isolated sites in particular.

\subsection{Challenges and expectations}

To guarantee an optimum surveillance level and to ensure that information is shared between the various parties concerned, CNR and SHEM need a powerful tool, for which parameters can be set easily and which is readily accessible. The main challenges were:

\footnotetext{
${ }^{a}$ Corresponding author: p.faure@cnr.tm.fr
} 
E1 To ensure the reliability of and to homogenise examination measurements

E2 To improve the examination measurement process with the help of ergonomic tools geared to needs

E3 To improve the analysis of measurements

E4 To homogenise and facilitate the graphics output

E5 To facilitate the monitoring of the structure's behaviour and decision-making

E6 To save time

E7 To improve the maintenance and the development of IT tools

\subsection{Scope}

The scope of the ALOHA project comprises the introduction of an IT tool covering:

- The management of equipment and sensors serving to examine hydraulic structures;

- The measurement validation workflow;

- The further use of measurements via analysis and graphics editing tools;

- The production of pre-formatted reports;

- The integration of transmitted data;

- Mobility solution for acquiring examination measurements including a fail-soft mode, where telecommunication is lost.

\subsection{The monitoring sensors}

The CNR and SHEM examination devices are largely identical and fairly similar to all the devices on the hydraulic structures. The park comprises mainly:

- $\quad$ Pendulums (absolute overturn and sliding);

- Vinchons - 3D fissurometers (differential movements: Upstream / Downstream, Right Bank / Left Bank, and compaction);

- Artesian and open piezometers (hydraulic loads);

- $\quad$ Level meters (of water surface);

- Flows; Topographical measurements (compaction, movements).

Each device is then fitted with one or more specific sensors allowing measurements to be transformed into physical components. These include:

- Front sight tables or tele-pendulums (pendulums);

- Vibrating wire cells;

- $\quad$ piezometric probe (open piezometers);
- $\quad$ Pressure gauges (artesian piezometers);

- Limnimetric scales (level meters).

Others monitoring systems are used by CNR or SHEM (topography, geophysics, drones, visual inspection etc..). However this are not, at present, managed by ALOHA.

\subsection{Measurement frequency}

In normal conditions of exploitation the frequencies of measurements vary from weekly to quarterly. In case of crisis, this resolution can decrease at hourly.

\section{DESCRIPTION REQUIREMENTS \\ OF \\ SPECIFIC}

As seen previously, the project brings together two independent concession-holders with structures having different characteristics and different operational methods. It was thus essential throughout the project to ensure that the needs of the two entities would be respected and that the solutions provided would be accepted by all users.

\subsection{Writing of functional specifications}

The project started with a feasibility study in late 2013 . The outcome was the decision to launch the complete redesign of the CASTOR examination software used in CNR.

The project started in early 2014 with 8 months of workshops with the users to write the functional specifications. Supported by a project assistance team, the group was able, in late 2014, to produce a functional specification covering all the functions wanted by users. This essential part of the project needed to be properly taken into account and correctly steered. Indeed, in a project of this scale, the involvement of future users is indispensable to take proper account of their functional needs and their ergonomic expectations. The success of the project depended largely on their proper integration in the upstream phase, i.e.: functional and technical responses, but above all appropriation by the users.

Following the consultation in late 2014, the development and integration project itself started in March 2015.

\subsection{Telemetry from the monitoring sensors}

SHEM's structures are dams that are independent of each other, each one having its own set of examination equipment, and references which differ from those of CNR (see 2.5).

Due to their situation in a highland and high mountain environment, SHEM's dams are subject to tough access constraints, particularly in winter. Thus, in order to overcome this physical isolation, much of the examination data for these dams does not have to be read at physical visits to the structures, but instead is transmitted directly to the database, via a telemetry device (collector) which forms the link between the examination equipment and ALOHA. 
The tool, therefore, needs to have an interface allowing the management of these telemetry data.

\subsection{Fail-soft mode}

For structures in isolated locations, losses of network connection can occur between the examination device and the application for processing the examination data. Continuity of service must nonetheless be guaranteed so as to be able to continue to acquire and validate these data while meeting requirements in terms of timing for level 1 and 2 analyses (see 3.1.5).

Thus, a specific module in the application which can be used on a mobile input terminal should allow the following situations to be managed:

- loss of the central unit (collector) that launches the telemetry

- loss of connection with the basic ALOHA tool.

Allowing the following actions to be carried out in another way:

- acquisition on the dam of data about the structure (on the mobile input terminal)

- offline validation of acquired examination data with, to this end, access to a sufficiently long history.

\subsection{Longitudinal sections and cross-sections}

Comprising 400 kilometres of permeable embankments, the CNR estate requires examination and thus a specific analysis. In addition to the water level chronicle from each of the piezometers, the engineers view and analyse the correct hydraulic function of the structure by means of views across or along the axis of the structure. The Cross-Sections (Figure 1) allow the correct hydraulic drawdown between the level of the Rhône and the level of the drainage canal to be viewed. The Longitudinal Sections for their part allow a check on the absence of zones of piezometric inconsistency in the longitudinal direction (piezometric level above or below its immediate neighbours).

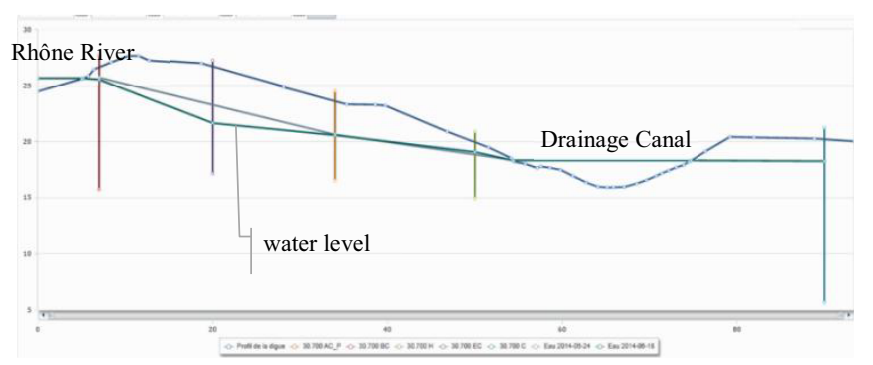

Figure 1: Example of a cross-section through the Right Bank dike at AVIGNON

\subsection{Linear references}

Due to its linear configuration along the River Rhône, the CNR concession has needed from the origin of the development studies in 1935, linear references from the French source of the Rhône to its mouth. The historic references have thus been Kilometric Points (KP) calculated from the confluence of the Saône and the Rhône in Lyon. Located in the direction of the sea and Lake Geneva, the KPs serve as references for positioning the piezometers and structures and for ship crews. The ALOHA project wanted from the outset, to use these reference points to position all the equipment and plant required for the examination work. From this PK information, the tool needs to be able to carry out water level calculations between two level meters or to link sets of sensors based on their linear positions.

\subsection{Validations and statuses}

To provide all users with the quality of examination data available in ALOHA, a certain number of statuses and business rules defining the acceptable system work flows had to be specified.

\subsubsection{Statuses in the application}

During the examination, the traceability and knowledge of the measurement quality is essential in order to be able to ensure that the behavioural analyses are based on correct and reliable measures. To guarantee this reliability to analysts, it was hoped that measurements, readings, measurement campaigns, components and observations could be connected to a sufficiently exhaustive and concise panel of statuses (Table 1). Depending on their status, the components will be visible to certain users and in specific symbologies.

\begin{tabular}{|l|l|l|}
\hline \multicolumn{2}{|c}{ Table 1: List of statuses } \\
\hline $\begin{array}{l}\text { Readings } \\
\text { measurements }\end{array}$ & $\begin{array}{l}\text { Awaiting } \\
\text { measurements }\end{array}$ & Not validated \\
\hline Measurement(s) & $\begin{array}{l}\text { Awaiting } \\
\text { Validation 1 }\end{array}$ & Validated 1 \\
\hline $\begin{array}{l}\text { Measurement(s) } \\
\text { confirmed }\end{array}$ & $\begin{array}{l}\text { Awaiting } \\
\text { Validation 2 }\end{array}$ & Validated 2 \\
\hline $\begin{array}{l}\text { Measurement(s) not } \\
\text { possible }\end{array}$ & $\begin{array}{l}\text { Under } \\
\text { consultation }\end{array}$ & Incorrect \\
\hline $\begin{array}{l}\text { Insignificant } \\
\text { components }\end{array}$ & & Doubtful \\
\hline
\end{tabular}

\subsubsection{Validation circuit}

Each component acquired by an examination unit must form the subject of two levels of analysis

- $\quad$ The 1 st level analysis (= validation 1$)$, which is to be carried out immediately or no later than 1.5 days after acquisition and consists of the visual identification of an abnormal measurement compared with the cloud of usual values for this unit. If needed, a return to site to check the instrument and a further measurement will allow the initial measurement to be confirmed or invalidated;

- $\quad$ The 2 nd level analysis (= validation 2), which aims to identify any abnormal measurement 
from the graph of raw components and then components at identical conditions; this must take place within 5 working days ( 1 day in the event of an earthquake or flood) following receipt by the competent expert of measurements from the 1 st level analysis.

ALOHA allows the completion of these two analysis levels to be traced thanks to the statuses described below.

In addition to the component validations, the $3^{\text {rd }}$ level analysis, consisting of a statistical analysis (HST) to detect the structure's behaviour over the long term, can be carried out outside of ALOHA.

\subsubsection{Graphics and scenarios}

The purpose of the examination measurements taken is to ensure that the structure's behaviour is monitored and appropriate decisions are made. In order to facilitate analysis by the engineers responsible for behavioural monitoring, ALOHA needed to provide a graphics module allowing the changes in the physical phenomena studied to be displayed based on variable scenarios. Thus, they needed to be capable of bringing together any of the various components by linking them taking into account their different parameters: uncertainty levels, trend graphs, thresholds etc.

These scenarios would then also be used to produce regulatory reports sometimes involving up to 150 different graphics. In this way, the operator would be able, in a single action, to produce all the "regulatory" graphics on the structure filtered in the application.

\subsection{Specification and strategy of flows}

Being a project carried by CNR and SHEM from the start, the flow strategy wanted by the SI CNR needed to allow exchanges between a variety of geographical sites and users. The basic premise was a dissociation in the production of the CNR and SHEM bases, although they would be hosted by CNR. SHEM and CNR users would then only be able access the base of their respective companies. In addition to internal users, the two bases needed to be open to external service providers involved in the behavioural analysis or level 2 validation (paragraph 2.6). The tool also had to be able to communicate easily with other proprietary databases, to retrieve telemetry data, associated measurements (hydrometry) or GIS data (base maps, land data etc.).

\section{PROJECT PROGRESS}

\subsection{Key points and risks associated with the project}

As the objective was to bring the solution into production in less than 10 months, the project phasing had to be optimised in order to meet the deadlines set (Figure 2).

The project team identified 5 project risks in its preliminary analysis:

\subsubsection{Non-compliance with the cut-off date for production of 24 December 2015}

The launch meeting for the ALOHA project was held on 16 March 2015. One of the objectives set at this meeting was to complete the specification workshops by the end of April. By the start of May, the following works had been carried out:

- Transfer of data from the Castor / SHEM / IGNHr bases with regard to the structures, equipment, sensors, photos, measurements, components, inspection routes, campaigns, scenarios and observations.

- Online launch of an initial version of ALOHA from early April. Regular updates throughout the project have allowed the new developments to be tested while "going with the flow" of the overall project schedule.

- Holding of user workshops for all the subjects initially planned.

- The interfaces with the other systems (central unit, GIS, etc.) had been specified.

- The functional specifications had been entirely revised to ensure that the various meetings covered all the points.

- The mobile ALOHA app had been specified.

One of the criteria for choosing the ScanPrint IMS ${ }^{\circledR}$ solution had been the level of cover of the functionalities proposed without specific development or settings. With around $70 \%$ of functionalities covered, it was possible to make use of the AGILE development method in order to validate the modules and developments as the project progressed.

\subsubsection{Understanding what CNR / SHEM needed}

The initial transfer of data allowed an initial version of ALOHA to be quickly put in place. Thus, it was possible from the start to organise user workshops on the solution under development, allowing needs to be specified and business rules to be described and adjusted in ScanPrint $\mathrm{IMS} \otimes$ in concrete terms.

These workshops took place over a 2-month period from March to May 2015. As the detailed specifications defined prior to the consultation were very detailed, the part analysing users' needs was thus relatively small and did not reveal any oversights or misunderstanding of what was needed.

\subsubsection{Acceptance of the solution by the end-users}

The initial presentations of ALOHA demonstrated that the application was intuitive and user-friendly. The endusers particularly appreciated certain functionalities:

- Quick access to data (quick search for a unit and direct access to the latest components)

- Display of units allocated to a tour of inspection in the cartography

- Reactivity of the solution

- Use of scenarios in the analysis 
- Filters

- Access to information about the units from various modules of the application

\subsubsection{Maintenance and upgradability}

The various workshops revealed that the ALOHA solution could adapt readily to needs not initially identified:

- Various types of observation

- Addition of new parameters for SHEM's sensors

- Addition of the concept of " reading", allowing orphan measurements without a campaign being carried out

- Adaptation of interfaces for workflow validation

\subsubsection{Performance of the solution}

The retrieval of data from the 3 bases provided has already allowed the system to be tested with operational data. Thus, it has been possible to carry out developments in order to optimise the performances of ALOHA to achieve consistent response times and offering enormous convenience of use.

\subsection{Architecture}

The solution relies on:

- a Microsoft SQL database server already present in the CNR's architecture. This is because the data of SHEM and CNR are split into 2 different bases.

- a dedicated IIS-hosted web application service (IIS : Internet Information Services)

The application server hosts the various applications for CNR and SHEM:

- The ALOHA Base: This is an RIA (Rich Internet Application) based on Entity Framework .NET and RIA services.

- ALOHA Mobile. This is an HTML5 app compatible with the various OS on the market (Windows, Windows Phone, Android and IOS). The app was developed on the Ionic and Angular JS frameworks.

The "https" protocol was put in place in order to ensure data security.

\subsection{Telemetry}

For the telemetry of dams in isolated sites, a single automatic examination data collection centre, the "Collector", connects at regular intervals to the local units specific to each dam (which are in turn connected to automatic measurement units fitted in the dams) and recovers the data acquired by the latter. The data from this collector must then be integrated in the ALOHA application. Thus a development specific to this need was carried out. ALOHA thus has a collector control interface (Figure 2) allowing agents to do the following directly from the application :
- Retrieve data collected at local unit level. During this retrieval operation, measurements and any error messages are consolidated in the general database. The measurement retrieval process is supplemented by the on-the-fly calculation of the various components.

- Control the acquisition cycle of each collector (frequency and date)

- Force an acquisition on a collector

\subsection{History retrieval}

The history retrieval was implemented essentially on 3 existing main bases:

- An Oracle base (Castor for CNR)

- An Access base (IGNHR for CNR)

- A series of Excel files (extraction of Panda data for SHEM).

The retrieval of a history is not simple a transfer of data from one data model to another. Indeed, data retrieval faced numerous problems:

- The various bases were managed differently from the measuring units. Thus, different mapping operations had to be performed to make the data consistent.

- The databases were not self-sustaining as certain business parameters were included in the applications. Thus, certain data also had to be retrieved in the applications.

- A verification of all the data was carried out during the retrieval process.

- As some data was incomplete, characterisation work was carried out in parallel.

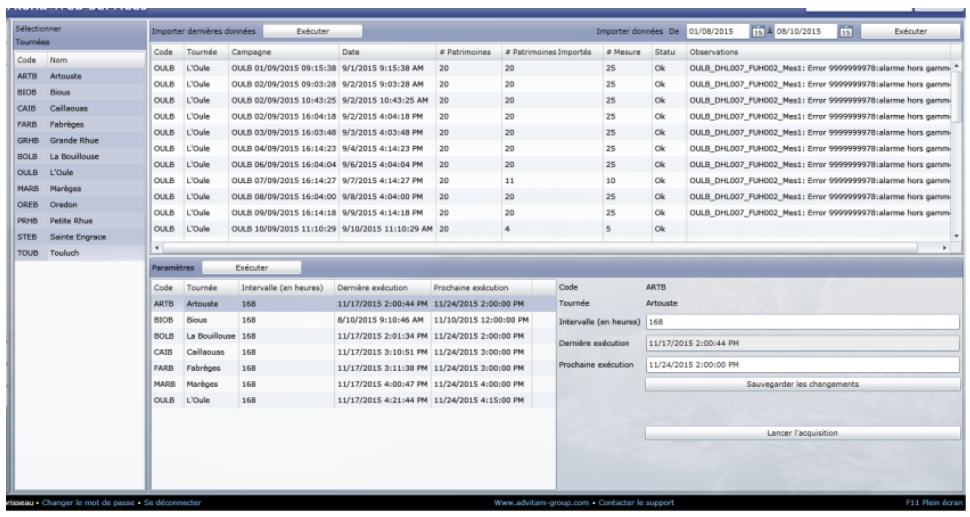

Figure 2: Collector control interface

\section{SPECIFIC FUNCTIONALITIES PROVIDED BY ALOHA}

\subsection{Property management}

While the systems used to date focus solely on the measurements of examination units, ALOHA allows a 
broader management of the issue and allows property management in the broad sense of the term.

Indeed, ALOHA includes a reference which contains all the assets concerned by the management cycle (powerplant, dams, locks, dikes, measuring equipment etc.) and also all the "objects" for organising this physical estate (the Rhône, the various canals, departments and developments). The linear referencing of CNR's devices explained in 2.5 means that the solution can rely on the reference axes (Right Bank and Left Bank) of the river and the Reference Points located at regular intervals (Figure 3).

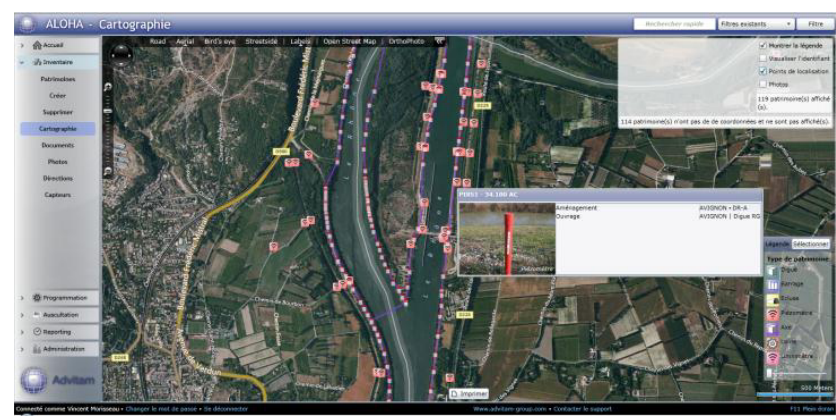

Figure 3: Illustration of the reference via the cartography interface

Thus, an "estate tree" was defined:

\section{The Rhône}

The VALLABREGUES development (between PK 232+000 and PK 266+000)

Beaucaire Power Station (at PK 254+533)

The Fissurometer VIN1 unit in the gallery at $1.20 \mathrm{~m}$.

The Fissurometer VIN2 unit in the gallery at $1.20 \mathrm{~m}$.

The Beaucaire Lock (at PK 254+364)

Left Bank Dike (between PK 232 and PK 266)

Piezometer CP1 unit (to PK 235+722)

Piezometer CP2 unit (to PK 235+950)

Right Bank Dike (between PK 232+000 and PK 266+000) $\cdots$

The AVIGNON development (between PK 216+000 and PK 232+000)

\subsection{Validation management}

The validation of the examination measurements is carried out in various stages (Figure 4):
- At data input, the operators need to confirm their measurements if the conformity tests carried out by the application are not verified. The tests verify the difference compared to the last measurement and that the measurement is not outside the range provided by the history;

- Once the measurements are confirmed, the data acquisition application calculates the components and presents them in a graph (2year history);

- Validation 1 is carried out by the operator, component by component using the graphics tools supplied by the application;

- Validation 2 is carried out by a third party (approver) who has a range of additional tools: validation scenarios, inter-component graphic, HST model.

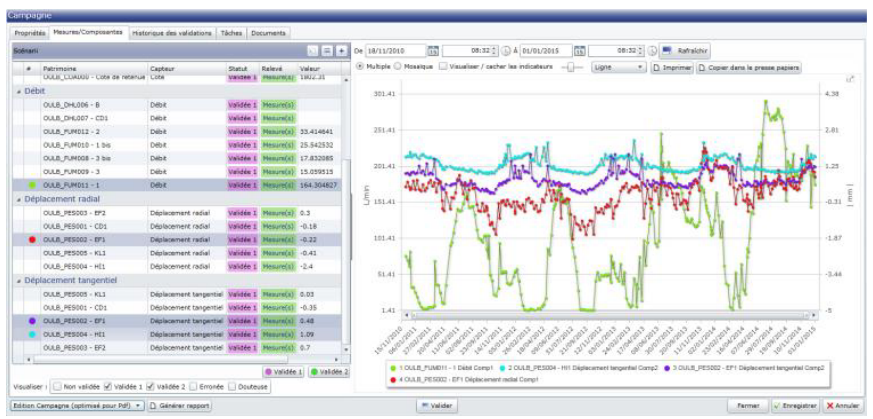

Figure 4: Validation of a campaign

\subsection{Graphics and scenarios}

The graphics were among the important project requirements as they are the medium for the behavioural analyses and constitute a large part of the regulatory deliverables.

To meet the requirements of users and facilitate their operation, the application's graphics module underwent specific development work so as to display the desired components with the help of predefined scenarios. This allows the following parameters to be set: the time range, titles, the components displayed and their respective ordinate axes and the display of threshold and trend graphics.

Being easy to set, select and modify, the scenarios can be used for graphical analyses, regulatory reports and validations.

A specific inter-component analysis interface (display of a component measures based on the results of another Figure 5) has also been developed to study seasonal effects. 


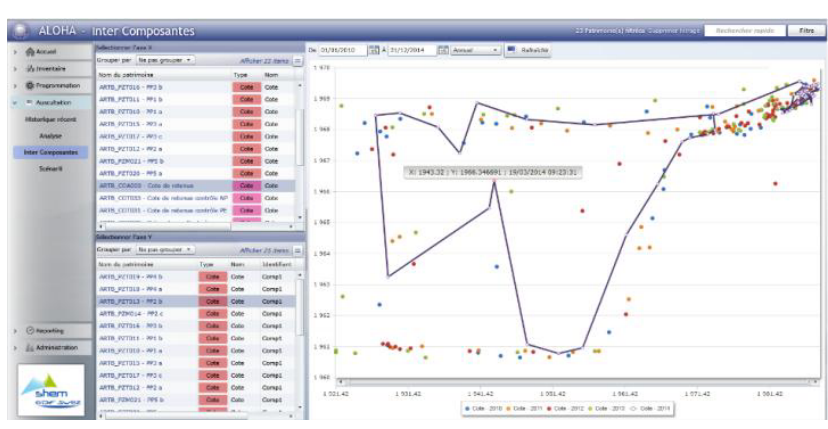

Figure 5: Inter-component management

\subsection{Mobility tool}

On the basis of ScanPrint Mobile, an interface was developed for inputting "readings". ALOHA Mobile allows readings and observations to be entered when working offline on tablets or smartphones.

The readings screen offers the following functionalities (Figure 6):

- Input of measurements;

- Carrying out of conformity tests on measurements;

- Calculation of components for recording measurements;

- Presentation of a 2-year history of components in graphics format;

- Display of general information about the reading (unit concerned, status);

- Addition of observations about the reading;

- Addition of photos;

- Addition of an anomalous finding.

The procedure for taking a reading follows the stages below:

- By default, the first sensor is selected, and one input field per measurement is accessible;

- For each measurement, the application states the name of the measurement, its unit and the 2 most recent values (history);

- As the user enters the measurements, the application carries out the 2 conformity tests. Each test is symbolised by a red or green shape;

- If one of the tests is not verified, the user must confirm the measurements: A "Confirm measurements" button appears, which the user then needs to click to be able to record the measurements;

- If measurements cannot be entered, the user can move on to the next sensor;

- In this case, the application automatically creates an anomalous finding in which the user enters additional information;

- Once a connection is possible on the ALOHA server, the reader synchronises the data, and the data is then available to everyone concerned.

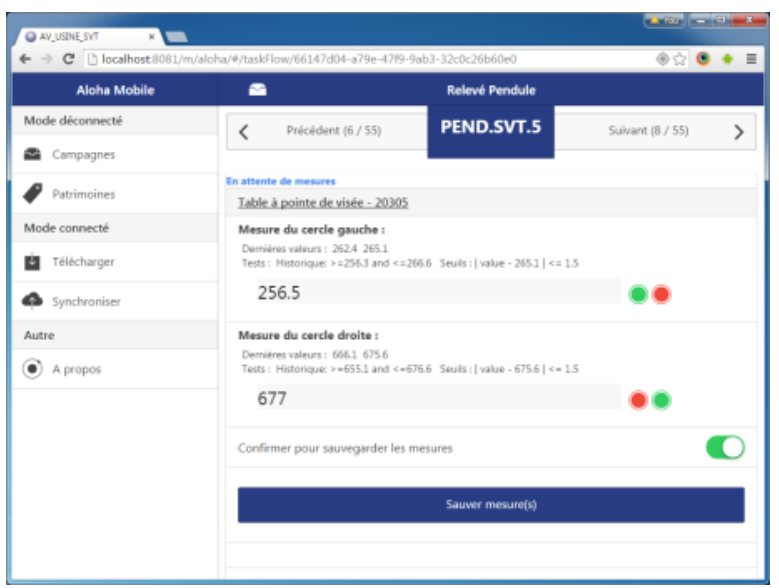

Figure 6: Illustrations of the mobile tool

\section{THE CONTRIBUTION OF THE ALOHA SOLUTION FOR RISK MANAGEMENT}

Good risk management is dependent on the ability of stakeholders to make the right decisions based on information from the field and the various tools helping the decision-making process. ALOHA is an integral part of the tools to help in decision-making in the face of a crisis due to its following characteristics:

- Integrated tool

- Workflow management

- Inter-operability and portability of tools

- Analysis tool

- Specific modules for the examination of hydraulic structures

\section{$5.1 \quad$ Integrated tool}

By its design and its various modules, ALOHA is an integrated solution in which a single interface is used to display all the information needed for the comprehensive management of the properties playing a part in hydraulic safety. By simple navigation, the user can move quickly from examination measurements to information about the structures (characteristics, operating constraints, associated documents etc.).

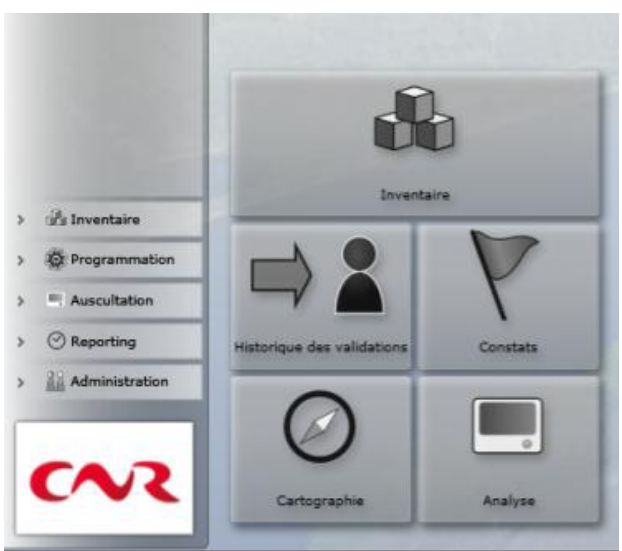

Figure 7: Interfaces to access the different modules 
Via a single interface, ALOHA allows the collection of the examination information (measurements) and information from visual inspections carried out in the field (Figure 8). Indeed, with the various modules, the field findings and the measures acquired by sensors can be collated for a single structure.
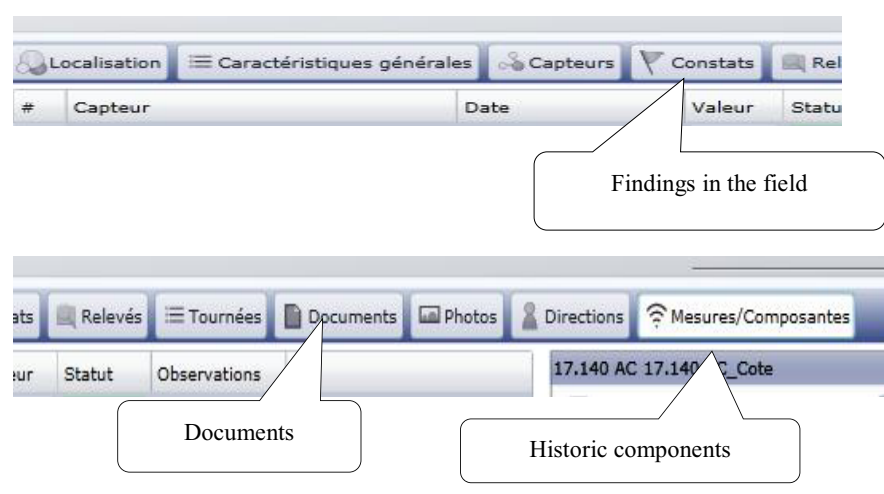

Figure 8: Illustration of access to the Findings, Document and Components module

\subsection{Workflow management}

ALOHA allows the various players involved in the risk management process to exchange information flows via various alerts and the monitoring of actions for which parameters can be set and which are diffused by electronic messaging.

Indeed, in addition to the possibility offered by the tool of putting in place vigilance or alert thresholds on the examination measurements, it also allows a workflow to be implemented between users to monitor findings.

\subsection{Portability of the solution to different hardware}

The full-web feature of ALOHA means that the ALOHA app can be deployed and used on any input terminal, be it a computer, a tablet or a smartphone. It is thus possible to make maximum use of the application without worrying about compatibility with the user's equipment.

When deploying the mobility aspect, the only constraint is that the terminal must have access to the base, at least at the start and end of the day to synchronise data. If the base is open on the internet, the operator can then, in real time, enter their measurements and findings from the field. This feature thus helps the right decisions to be reached in a minimum of time.

\subsection{Inter-operability and connection to external data}

Developed for concession-holders generating hydraulic electricity, the ALOHA tool is fully inter-operable with all business databases. These connections provide access from ALOHA to certain external information, for example:

- Maintenance management base;
- Operational management database;

- Production data;

- Hydrometry data;

- Estate data managed by other applications.

Moreover, the tool allows any type of flow of mapping data from local GIS or external open-data to be integrated.

\subsection{Specific modules for the examination of earth-fill structures}

The final important element for risk management and more particularly for flood risk management, is found in the modules dealing specifically with embankments. Due to its concession and its structures, CNR has specific needs with regard to the monitoring of permeable earthfill structures.

\subsubsection{Longitudinal sections and cross-sections}

With the interface for longitudinal sections and crosssections, the user can view in graphics form the correct hydraulic drawdown in the dam body (see Figure 1: Example of a cross-section through the Right Bank dike at AVIGNON). In addition to this graphics view, a "remaining load" component is calculated at the section in order to check that the drawdown is correct for the zone concerned.

These two tools, in combination with real-time feedback from the field (automatic and manual measurements can be sent in real time from the field) provide the manager with indicators about the state of the structure and thus objective criteria for the management of the risk.

\subsubsection{Management of stretches of embankments}

When the tool was introduced and as seen in paragraph 4.1, a reference base structured around reference axes (Right Bank and Left Bank of the Rhône) and kilometre Reference Points was created over the entire CNR concession. Thanks to this reference base, it is now possible to locate (Figure 9), automatically draw and manage information concerning all the stretches of the CNR's embankments (approx. 340 over $400 \mathrm{~km}$ ). 


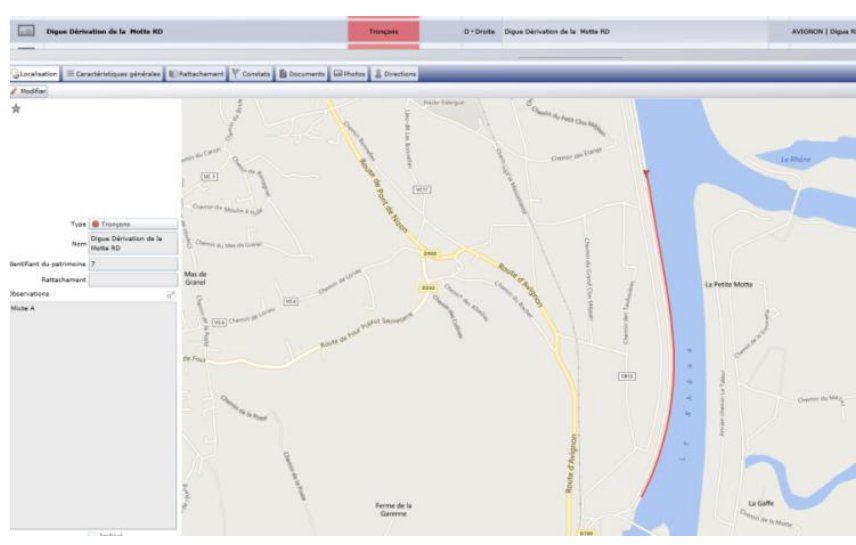

Figure 9: Location of a stretches on the Avignon development

\section{FEW FIGURES}

- $15000(\mathrm{CNR})+500($ SHEM) devices

- 300000 (CNR) + 10000 (SHEM) attribute fields

- $75000(\mathrm{CNR})+3300$ (SHEM) sensors and Component.

- 29000 (CNR) + 19000 (SHEM) historical campaign.

- 1700000 (CNR) + 278000 (SHEM) historical Readings

- 3800000 (CNR) + 700000 (SHEM) historical measurements and component

- 8500 (CNR) photographys and documents

- 11000 (CNR) reference points (PR)

- $30000(\mathrm{CNR})$ finding field and comments

\section{CONCLUSION AND FURTHER ACTION}

To support the advent of new technologies and changes to methods and regulatory requirements, $\mathrm{CNR}$ and SHEM, because of their proximity (common shareholder) have come together to develop a new dedicated tool for the monitoring and surveillance of structures. Based on often shared needs, sometimes very specific to their structures and organisation, the two businesses were able to define a detailed functional specification. Responding to a call for tenders, the company, Advitam-group, provided a solution based on its ScanPrint IMS ${ }^{\circ}$ package. As it did not offer all the desired functionalities, the Advitam-group developed innovative specific modules:

- $\quad$ specific mobile tool and functionalities

- concept of readings allowing measurements, components and observations to be attached to an examination device;
- concept of scenarios for validations and production of reports

The functionalities available in ALOHA V1 at the end of 2015 cover $95 \%$ of the needs described by the users at the start of the project and offer possibilities not initially planned. Feedback is positive overall while awaiting a version 2 of the tool in 2016 in order to cover $100 \%$ of the business functionalities.

The project team, want to focus on the production of a functional specification covering all the functions wanted by user. This part is essential and to be properly taken into account and correctly steered. Indeed, in a project of this scale, the involvement of future users is indispensable to take proper account of their functional needs and their ergonomic expectations. The success of the project depended largely on their proper integration in the upstream phase, i.e.: functional and technical responses, but above all appropriation by the users.

\section{ACKNOWLEDGEMENTS}

The authors thank CNR, SHEM and Advitam-Group for their support during the project. A special thanks also to all the users having participated in the writing of the specifications or in the receipts. 